\title{
Nomen est omen - über die Namensgebung bei Pflanzen
}

\author{
Peter Gausmann \& GÖTZ HeInRICH LoOs
}

\begin{abstract}
Plant systematics and taxonomy are connected with botanical nomenclature. The scientific names of genera and species often honour famous botanists. Recently, a new genus Haeupleria was described. It is named after HeNNING HaEUPLER, a botanist from the universities in Göttingen and Bochum.
\end{abstract}

\section{Zusammenfassung}

Systematik und Taxonomie stehen in Bezug zur wissenschaftlichen Benennung von Pflanzen. Gattungs- und Artnamen ehren oft berühmte Botaniker. In jüngster Zeit wurde die neue Gattung Haeupleria für ein Gras aufgestellt. Sie ehrt den Göttinger und Bochumer Geobotaniker HeNNING HAEUPLER.

\section{Systematik, Taxonomie und Nomenklatur} Taxonomie, Systematik und Nomenklatur sind Teil-Disziplinen der Botanik, die sich mit Identifizierung, Beschreibung und Benennung von Pflanzenarten befassen. Die Pflanzensystematik versucht, eine den natürlichen Verwandtschaftsbeziehungen entsprechende Klassifikation zu liefern. Heute liefert dabei die Analyse des Erbgutes (DNA) entscheidende Hinweise.

Ganz wesentlich ist nach wie vor die Nomenklatur, wobei jeder Pflanze ein eindeutiger wissenschaftlicher Namen zugeordnet wird. Dadurch wird die Kommunikation über Pflanzen in unterschiedlichen Sprachräumen vereinfacht. Volksnamen sind nicht immer eindeutig bestimmten Pflanzen zuzuordnen, wie das bekannte Beispiel der Butterblume, unter der man sowohl Ranunculus acris als auch Vertreter der Gattung Taraxacum verstehen kann.

Die wissenschaftliche Benennung einer Pflanzenart erfolgt nach weltweit einheitlichen Kriterien, die im Internationalen Code der Botanischen Nomenklatur (ICBN, aktuelle Fassung bei MCNeILL et al. 2006) festgelegt sind. Für Kulturpflanzen und deren zahlreich vorhandene Sortennamen wird der ICBN durch den Internationalen Code der Nomenklatur der Kulturpflanzen ergänzt (ICNCP, ISHS 2009).

Der wissenschaftliche Name einer Pflanzenart setzt sich aus zwei Teilen zusammen, dem Gattungsnamen und dem Art-Beinamen (Epitheton). Weil die Namen aus zwei Teilen bestehen, spricht man auch von Binomen. Die wissenschaftlichen Namen sind zumeist lateinischen oder griechischen (und dann oft latinisierten) Ursprungs, können aber auch auf andere Sprachen zurückgehen. Der wissenschaftliche Name beruht immer auf einer gültigen Veröffentlichung, in welcher eine Beschreibung und Benennung erfolgt. Nach den Kriterien des ICBN gehört dazu auch heute noch eine in lateinischer Sprache verfasste Beschreibung (Diagnose) der jeweiligen Sippe (Art, Gattung, Unterart etc.) sowie die Ausweisung und Angabe eines TypusBeleg-Exemplares, das in einem Herbarium einer Forschungseinrichtung oder in einem Museum hinterlegt wird.

Die heute übliche binäre Nomenklatur geht auf den schwedischen Naturforscher CARL VON LiNNÉ (*1707, †1778) und sein botanisches Hauptwerk „Species Plantarum“ (1753) zurück. Das hinter vielen Arten stehende „L.“ ist das Autorenkürzel für LINNÉ. Die wörtliche Übersetzung des wissenschaftlichen Namens lässt oft Schlüsse auf das Aussehen oder die Verwendung der Pflanze zu. So bedeutet z. B. der Art-Beiname nucifera für die Kokosnuss nichts anders als nüssetragend.

\section{Personen in wissenschaftlichen Namen}

Bisweilen werden aber auch Forscher, andere berühmte Persönlichkeiten, Dichter, Adelige, Heilige oder Gestalten aus der griechischen Mythologie in den Namen verewigt (SEYBOLD et al. 2008, MABBERLEY 2008). So ist beispielsweise der wissenschaftliche Name der Paradiesvogelblume (Strelitzia reginae) doppelt königlich. Der Gattungsname ehrt die britische Königin 

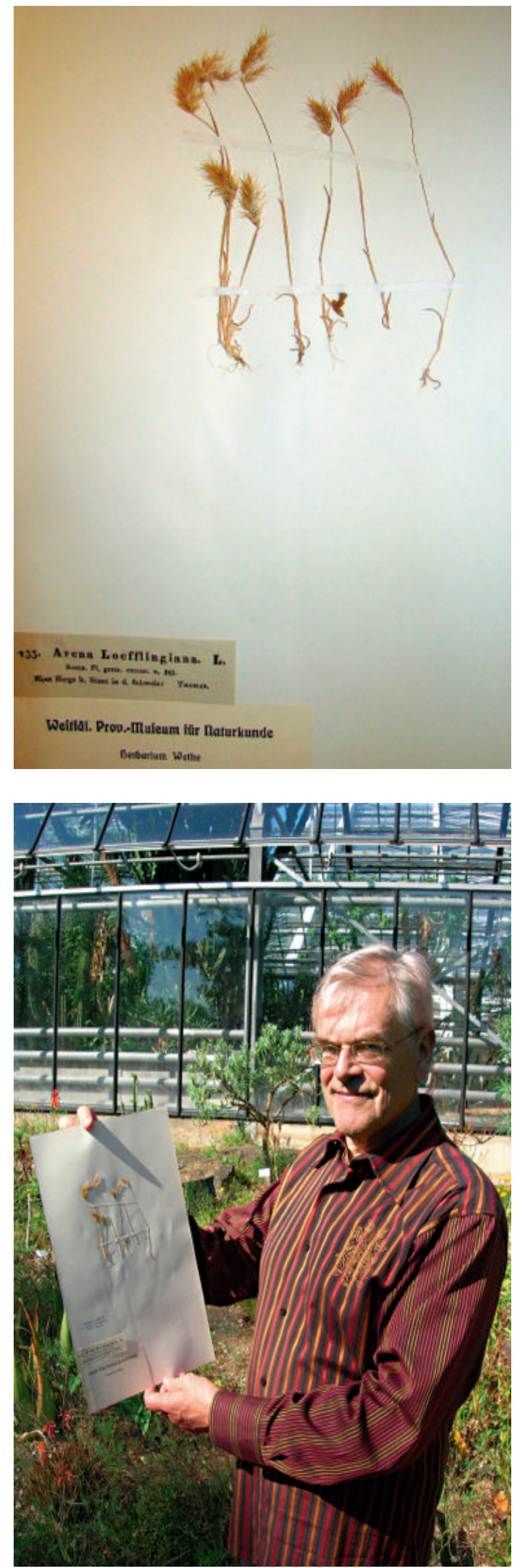

Charlotte, eine geborene Prinzessin von Mecklenburg-Strelitz und Gemahlin Königs Georg III. Die lateinische Vokabel regina bedeutet Königin.

Botaniker werden bei der Vergabe von Gattungs- oder Artnamen gerne verewigt, aber auch z. B. ehemalige Lehrer, Freunde, Lebenspartner oder gar Gegner. Ein Beispiel für die Ehrung eines Botanikers ist der Name des in Mitteleuropa als Neophyt eingebürgerter und als giftiger Eindringling gefürchteter Riesen-Bärenklau ( $\mathrm{He}$ racleum mantegazzianum SOMMIER \& LEVIER). Sein Art-Beiname geht auf den italienischen Botaniker Paolo Mantegazzi $(* 1831, \dagger 1910)$ zurück. Wird ein Eigenname im Art-Beinamen verwendet, steht dort oft der Genitiv des latinisierten Familien-Namens. Beispielsweise wurde nach HermanN WildPret, einem Schweizer Gärtner und Botaniker aus dem 18. Jh., der sich intensiv mit der Kanaren-Flora befasste, Wildprets Natternkopf (Echium wildpretii) benannt. Dem Dichterfürsten GOETHE wurde bereits zu Lebzeiten die Gattung Goethea (Malvaceae) gewidmet.

\section{Zeitgenössische Botaniker und neue Gattungsnamen}

Da neue Gattungen heutzutage erheblich seltener aus der Taufe gehoben werden als Arten, sind aktuelle Gattungs-Benennungen nach zeitgenössischen Botanikern selten. Allerdings geben Ergebnisse aus DNA-Sequenzanalysen immer wieder neue Hinweise auf sinnvolle Abgrenzungen einzelner Gattungen und erfordern gelegentlich Gattungs-Neubeschreibungen.

Ein Beispiel für die Anlehnung des Gattungsnamens an Botaniker aus der jüngsten Zeit ist Haeupleria. Der Zweitautor widmete diese heimische Gras-Gattung seinem Lehrer, dem Göttinger und Bochumer Geobotaniker HeNnING E. Haeupler zum 70. Geburtstag (vergl. LoOS

Abb. 1 (oben): Herbarbeleg von Haeupleria loefflingiana.

Abb. 2 (unten): HenNing HaEUPLER mit „seiner“ Haeupleria. HAEUPLER gilt als Begründer der Floristischen Kartierung Deutschlands auf Messtischblatt-Basis. In seiner Göttinger Zeit leistete er v. a. wichtige Beiträge zur Erforschung der süd-niedersächsischen Flora, über die er auch promovierte. 
2010). HAEUPLER, mittlerweile Emeritus, leitete fast 25 Jahre lang die AG Geobotanik des Lehrstuhls für Evolution und Biodiversität der Pflanzen an der Ruhr-Universität Bochum. HAEUPLER wurde in Botanikerkreisen u. a. durch seinen Verbreitungsatlas der Farn- und Blütenpflanzen Deutschlands, später auch speziell NordrheinWestfalens sowie den dazugehörigen Bildatlas bekannt (HAEUPLER \& SCHÖNFELDER 1988, Haeupler \& Muer 1997, Haeupler et al. 2003).

Haeupleria wurde aufgrund von Befunden aus DNA-Sequenzierungen, kombiniert mit äußerlichen Merkmalen, von der großen, in sich sehr vielgestaltigen Gattung Trisetum (Goldhafer) abgetrennt (vgl. Loos 2010). Die Untersuchungen haben gezeigt, dass nicht allen Arten der Gattung Trisetum dieselbe Evolutionslinie zugrunde liegt. Eine Abtrennung und Einbeziehung in die nächst verwandte Gattung Gaudinia erschien aufgrund morphologischer Unterschiede nicht sinnvoll, weshalb die neue Gattung Haeupleria eingerichtet wurde. Gräser der Gattung Haeupleria sind einjährig und werden etwa 15-20 Zentimeter hoch. Ihr dichter Blütenstand ist eine ovale Rispe. Die Typus-Art ist Haeupleria ovata (früher Trisetaria ovata), eine westmittelmediterrane, hauptsächlich in Spanien vorkommende Art. Außerdem umfasst die Gattung noch $H$. cavanillesii und $H$. loeflingiana, zwei sehr nahe miteinander verwandte Arten, von denen die erstgenannte in den Alpen zu finden ist, $H$. loeflingiana wiederum in Spanien.
Noch weitere unklare Arten des TrisetumKomplexes müssten in Zukunft genauer erforscht werden, um sie besser gegenüber Trisetum im engeren Sinne abgrenzen zu können.

\section{Dank}

Herrn Dr. BeRnd TenBERGEN vom Landschaftsverband Westfalen-Lippe (LWL) und Herbarium Münster danken wir für die zügige Übersendung der Herbar-Belege.

\footnotetext{
Literatur

Haeupler, H. \& Schoenfelder, P. 1988: Atlas der Farnund Blütenpflanzen der Bundesrepublik Deutschland. Stuttgart.

Haeupler, H., Jagel, A. \& Schumacher, W. 2003:

Verbreitungsatlas der Farn- und Blütenpflanzen in Nordrhein-Westfalen. - Recklinghausen.

Haeupler, H. \& Muer, T. 2007: Bildatlas der Farn- und Blütenpflanzen Deutschlands. 2. Aufl. - Stuttgart. INTERNATIONAL SOCIETY FOR HORTICULTURAL SCIENCE (ISHS) 2009: International code of nomenclature for cultivated plants. - Scripta Horticulturae 10.

Loos, G. H. 2010: Taxonomische Neukombinationen zur Flora Mittel- und Osteuropas, insbesondere NordrheinWestfalens. - Jahrb. Bochumer Bot. Ver. 1: 114-133. MABBERLEY, D. J. 2008: Mabberley's plant book. A portable dictionary of plants, their classifications and uses. 3. Aufl. - Cambridge.

MCNeILl, J. (Hrsg.) 2006: International code of botanical nomenclature (Vienna Code). - Regnum Veg. 146.

SEYBOLd, S., BÖDEKER, N., EHRHARDT, W. \& GÖTZ, E. 2008:

Der große Zander. Enzyklopädie der Pflanzennamen. Stuttgart.
} 